\title{
The dimensionality of functional independence measure (FIM)
}

\begin{abstract}
This is an audit of the dimensionality of FIM referring to a Ravaud et al. ${ }^{1}$ study in which was indicated that the use of FIM scores is not justified because the assumption of unidimensionality is not given. Performing a factor analysis with our FIM assessment data we conclude that although the two-dimension solution holds for many data sets, other possibilities should be considered as changes of dimensionality are also empirical results. A search for valid instruments based on phenomenological, ecological and contextual conceptualization of independence is encouraged.
\end{abstract}

Volume 3 Issue 5 - 2018

Valach Ladislav, Selz Beat
Designation University, Switzerland

Correspondence: Ladislav Valach, PhD, Designation

University, Lindenstrasse 26, 3047 Bremgarten, Switzerland, Tel +31792162192, Email ladislav.valach@swissonline.ch

\section{Introduction}

The widespread practice of adding all values of the 18 FIM items into an overall score has been occasionally questioned. Although the FIM items correlate highly among themselves and the reliability coefficient alpha is very high, the issue of the linearity, interchangeability, equal distances etc. is still open. Ravaud et al. ${ }^{1}$ warned of using the FIM total score without precaution because of the multidimensionality of the FIM items. They recommend using various subscores. Though this is a sensible suggestion it is quite possible that the dimensionality of FIM will vary in different languages, in different age groups, illnesses etc. Consequently, before FIM is used as an idiographic measure in individual diagnostics, a dimensional analysis of the relevant data (language, diagnosis etc.) should be performed. This also is a task of test construction procedure whenever test is used in different languages, for different patients etc. This discussion continues. ${ }^{2}$

The purpose of this paper is to describe the dimensionality of FIM as used in the Rehabilitation Clinic in Solothurn (RGM BSS).

\section{Method}

The Functional Independence Measure (FIM) is an 18 item observational sheet and interview schedule. Each of the patient's activities (self care, sphincter management, transfer, locomotion, communication and social cognition) is rated between 1 (totally dependent) and 7 (fully independent). The FIM was filled out for every patient treated at the Department of Rehabilitation and Geriatric Medicine of the Buerger Hospital in Solothurn during 1998 ( $\mathrm{N}=250)$. Additional personal and socio demographic data were also collected. This evaluation contains data of the observations made on admission and on discharge of the patients.

The patients were either post surgery $(22.1 \%)$, or suffered vascular brain damage $(30.4 \%)$, traumatic brain damage $(2.5 \%)$, rheumatologic illness $(16.4 \%)$, geriatric multi organ illness $(8.2 \%)$ or other neurological illnesses (19.3\%). There were $49.3 \%$ women, $50.7 \%$ men, mean age $65.3(\mathrm{sd}=16.5)$.

\section{Results}

The factor analysis of all patients $(\mathrm{N}=250)$ indicates that the often reported two dimension solution (Factor 1: motor and Factor
2: cognitive items) is easily reproducible (Table 1) (Table 2) (Table 3 ). These two factors explain about $78 \%$ of the variance which is a high proportion. The factor analysis of the FIM on admission and on discharge do not differ substantially. A factor analysis of the 13 motor items alone provides a one factor solution which explains about $75 \%$ of the variance (Table 4) (Table 5). Again, the factor analyses of the FIM items on admission and on discharge do not differ significantly, the lowest loading is reached by the item 'Eating' and the highest by the transfer items (bed/chair, toilet, tub, in the case of FIM on admission, and bed/chair and toilet in the case of discharge).

Table I Diagnosis

\begin{tabular}{ll}
\hline Diagnosis & $\mathbf{N}$ \\
\hline vascular brain damage & 75 \\
traumatic brain damage & 9 \\
post surgery & 56 \\
rheumatologic illness & 44 \\
geriatric multi organ illness & 20 \\
other neurological illnesses & 46 \\
all & 250 \\
\hline
\end{tabular}

Table 2 Factor analysis of FIM on admission, all patients $(\mathrm{N}=250)$ (extraction by principal component analysis)

\begin{tabular}{llll}
\hline Factor & Eigenvalue & $\%$ of variance & $\begin{array}{l}\text { cumulative } \\
\%\end{array}$ \\
\hline $\mathrm{I}$ & $1 \mathrm{l} .69$ & 64.9 & \\
2 & 2.34 & 13 & 77.9 \\
\hline $\begin{array}{l}\text { Rotated factor matrix } \\
\text { (Varimax) }\end{array}$ & Factor I & Factor 2 \\
\hline $\begin{array}{l}\text { Self care: } \\
\text { A. Eating }\end{array}$ & 0.61 & 0.49 \\
B. Grooming & & \\
\hline
\end{tabular}


Table continued..

\begin{tabular}{|c|c|c|c|c|c|c|}
\hline \multirow{2}{*}{$\begin{array}{l}\text { Rotated factor matrix } \\
\text { (Varimax) }\end{array}$} & \multirow{2}{*}{ Factor I } & \multirow{2}{*}{ Factor 2} & & & & \\
\hline & & & Factor & Eigenvalue & $\%$ of variance & $\begin{array}{l}\text { cumulative } \\
\%\end{array}$ \\
\hline C. Bathing & 0.84 & 0.28 &  & 11.75 & 65.2 & \\
\hline $\begin{array}{l}\text { D. Upper body } \\
\text { dressing } .76\end{array}$ & & 0.43 & 2 & 2.19 & 12.2 & 77.4 \\
\hline $\begin{array}{l}\text { E. Lower body } \\
\text { dressing } .88\end{array}$ & & 0.22 & \multicolumn{2}{|l|}{$\begin{array}{l}\text { Rotated factor matrix } \\
\text { (Varimax) }\end{array}$} & Factor I & Factor 2 \\
\hline F.Toiletting & 0.86 & 0.29 & \multicolumn{2}{|l|}{ Self care: } & 0.66 & 0.43 \\
\hline $\begin{array}{l}\text { Sphincter } \\
\text { control: }\end{array}$ & 0.74 & 0.4 & \multicolumn{2}{|l|}{ A. Eating } & & \\
\hline G. Bladder & & & \multicolumn{2}{|l|}{ B. Grooming } & 0.74 & 0.45 \\
\hline management & & & \multicolumn{2}{|l|}{ C. Bathing } & 0.86 & 0.27 \\
\hline $\begin{array}{l}\text { H. Bowel } \\
\text { management }\end{array}$ & 0.7 & 0.43 & \multicolumn{2}{|l|}{$\begin{array}{l}\text { D. Upper body } \\
\text { dressing } .77\end{array}$} & & 0.44 \\
\hline Transfer: & 0.9 & 0.24 & \multicolumn{2}{|l|}{$\begin{array}{l}\text { E. Lower body } \\
\text { dressing } .87\end{array}$} & & 0.22 \\
\hline & & & \multicolumn{2}{|l|}{ F.Toiletting } & 0.85 & 0.33 \\
\hline \multicolumn{2}{|l|}{ I. Bed/chair/WC } & & \multicolumn{2}{|l|}{ Sphincter control: } & 0.74 & 0.34 \\
\hline J.Toilet & 0.91 & 0.22 & \multicolumn{2}{|l|}{$\begin{array}{l}\text { G. Bladder } \\
\text { management }\end{array}$} & & \\
\hline K.Tub/shower & 0.92 & 0.19 & \multicolumn{2}{|l|}{$\begin{array}{l}\text { H. Bowel } \\
\text { management }\end{array}$} & 0.72 & 0.37 \\
\hline Locomotion: & 0.82 & 0.29 & \multicolumn{2}{|l|}{ Transfer: } & 0.91 & 0.22 \\
\hline & & & \multicolumn{3}{|l|}{ I. Bed/chair/WC } & \\
\hline \multicolumn{3}{|l|}{ L.Walk/WC } & \multicolumn{2}{|l|}{ J.Toilet } & 0.91 & 0.23 \\
\hline M. Stairs & 0.84 & 0.1 & \multicolumn{2}{|l|}{ K.Tub/shower } & 0.89 & 0.23 \\
\hline Communication: & 0.18 & 0.91 & $\begin{array}{l}\text { Locomotion: } \\
\text { L.Walk/WC }\end{array}$ & & 0.82 & 0.26 \\
\hline \multicolumn{7}{|l|}{$\mathrm{N}$} \\
\hline Comprehension & & & \multicolumn{2}{|l|}{ M. Stairs } & 0.81 & 0.16 \\
\hline O. Expression & 0.18 & 0.87 & \multicolumn{2}{|l|}{ Communication: } & 0.21 & 0.88 \\
\hline & & & \multicolumn{3}{|l|}{ N. Comprehension } & \\
\hline Social cognition: & 0.23 & 0.84 & \multicolumn{2}{|l|}{ O. Expression } & 0.25 & 0.85 \\
\hline $\begin{array}{l}\text { P. Social } \\
\text { interaction }\end{array}$ & & & Social cognition: & & 0.2 & 0.83 \\
\hline Q. Problem & 0.43 & 0.78 & P. Social interaction & & & \\
\hline & & & Q. Problem solving & & 0.41 & 0.8 \\
\hline R. Memory & 0.31 & 0.85 & R. Memory & & 0.31 & 0.86 \\
\hline
\end{tabular}


Table 4 Factor analysis of 13 motor FIM items on admission, all patients $(\mathrm{N}=250)$

\begin{tabular}{lll}
\hline Factor & Eigenvalue & \% of variance \\
\hline I & 9.78 & 75.2 \\
\hline & Factor I & \\
\hline Self care: & 0.76 \\
A. Eating & \\
B. Grooming & 0.86 \\
C. Bathing & 0.89 \\
D. Upper body dressing & 0.87 \\
E. Lower body dressing & 0.89 \\
F.Toiletting & 0.9 \\
Sphincter control: & 0.83 \\
G. Bladder management & \\
H. Bowel management & 0.81 \\
Transfer: & 0.92 \\
I. Bed/chair/WC & \\
J.Toilet & 0.92 \\
K. Tub/shower & 0.92 \\
Locomotion: & 0.87 \\
L.Walk/WC & \\
M. Stairs & 0.81 \\
\hline
\end{tabular}

Table 5 Factor analysis of 13 motor FIM items on discharge, all patients $(\mathrm{N}=250)$

\begin{tabular}{lll}
\hline Factor & Eigenvalue & \% of variance \\
\hline I & $9.8 \mathrm{I}$ & 75.5 \\
\hline & Factor I & \\
\hline Self care: & 0.78 \\
A. Eating & \\
B. Grooming & 0.86 \\
C. Bathing & 0.9 \\
D. Upper body dressing & 0.89 \\
E. Lower body dressing & 0.89 \\
F.Toiletting & 0.91 \\
Sphincter control: & 0.82 \\
G. Bladder management & \\
H. Bowel management & 0.81 \\
Transfer: & 0.92 \\
I. Bed/chair/WC & \\
J.Toilet & 0.92 \\
K.Tub/shower & 0.9 \\
Locomotion: & 0.86 \\
L.Walk/WC & \\
M. Stairs & \\
\hline
\end{tabular}

Although some authors maintain that factor analysis should be performed only with a sample about 10 times larger than the amount of variables, others indicate that this does not make any difference. We attempted factor analyses with smaller samples while analysing the patients with a specific diagnosis and considering these results as preliminary and exploratory.

A factor analysis of the patients with vascular brain damage $(\mathrm{N}=75)$ provided a two-factor solution comparable to the whole sample of patients (Factor 1: motor and Factor 2: cognitive items). These solutions did not differ for the FIM items on admission and on discharge (Table 6) (Table 7) (Table 8). The factor analytical solution of the 13 FIM motor item did not differ from the results obtained in the analysis of the whole sample on admission and on discharge.

Table 6 Factor analysis of FIM on admission, vascular brain damage patients $(\mathrm{N}=75)$

\begin{tabular}{|c|c|c|c|}
\hline Factor & Eigenvalue & $\%$ of variance & cumulative $\%$ \\
\hline I & 12.51 & 69.5 & 79.8 \\
\hline 2 & 1.87 & 10.4 & \\
\hline \multicolumn{2}{|c|}{$\begin{array}{l}\text { Rotated factor matrix } \\
\text { (Varimax) }\end{array}$} & Factor I & Factor 2 \\
\hline \multicolumn{2}{|l|}{ Self care: } & 0.64 & 0.52 \\
\hline \multicolumn{4}{|l|}{ A. Eating } \\
\hline \multicolumn{2}{|l|}{ B. Grooming } & 0.71 & 0.56 \\
\hline \multicolumn{2}{|l|}{ C. Bathing } & 0.76 & 0.43 \\
\hline \multicolumn{2}{|l|}{ D. Upper body dressing } & 0.74 & 0.5 \\
\hline \multicolumn{2}{|l|}{ E. Lower body dressing } & 0.87 & 0.28 \\
\hline \multicolumn{2}{|l|}{ F.Toiletting } & 0.89 & 0.31 \\
\hline \multicolumn{2}{|l|}{ Sphincter control: } & 0.79 & 0.4 \\
\hline \multicolumn{4}{|l|}{ G. Bladder management } \\
\hline \multicolumn{2}{|l|}{ H. Bowel management } & 0.72 & 0.5 \\
\hline \multicolumn{2}{|l|}{ Transfer: } & 0.91 & 0.28 \\
\hline \multicolumn{4}{|l|}{ I. Bed/chair/WC } \\
\hline \multicolumn{2}{|l|}{ J.Toilet } & 0.92 & 0.27 \\
\hline \multicolumn{2}{|l|}{ K.Tub/shower } & 0.92 & 0.23 \\
\hline \multicolumn{2}{|l|}{ Locomotion: } & 0.81 & 0.36 \\
\hline \multicolumn{4}{|l|}{ L.Walk/WC } \\
\hline \multicolumn{2}{|l|}{ M. Stairs } & 0.89 & 0.12 \\
\hline \multicolumn{2}{|l|}{ Communication: } & 0.16 & 0.9 \\
\hline \multicolumn{4}{|l|}{ N. Comprehension } \\
\hline \multicolumn{2}{|l|}{ O. Expression } & 0.17 & 0.84 \\
\hline \multicolumn{2}{|l|}{ Social cognition: } & 0.3 & 0.76 \\
\hline \multicolumn{4}{|l|}{ P. Social interaction } \\
\hline \multicolumn{2}{|l|}{ Q. Problem solving } & 0.53 & 0.73 \\
\hline \multicolumn{2}{|l|}{ R. Memory } & 0.38 & 0.76 \\
\hline
\end{tabular}


Table 7 Factor analysis of FIM on discharge, vascular brain damage patients $(N=75)$

\begin{tabular}{llll} 
Factor & Eigenvalue & $\%$ of variance & cumulative \% \\
\hline 1 & 12.43 & 69 & \\
2 & 1.94 & 10.8 & 79.8
\end{tabular}

Rotated factor matrix
(Varimax)

Factor I $\quad$ Factor 2

Self care:

0.74

0.34

A. Eating

B. Grooming

0.75

\section{Bathing}

0.82

D. Upper body dressing .75

E. Lower body dressing .89

F. Toiletting

0.88

0.37

Sphincter control:

0.81

0.24

G. Bladder management

H. Bowel management

Transfer:

I. Bed/chair/WC

J.Toilet

K.Tub/shower

Locomotion:

L.Walk/WC

M. Stairs

Communication:

0.21

0.88

N. Comprehension

O. Expression

Social cognition:

0.2

0.79

P. Social interaction

Q. Problem solving

0.5

0.73

R. Memory

0.37
Table 8 Factor analysis of 13 motor FIM items on admission, vascular brain damage patients $(\mathrm{N}=75)$

\begin{tabular}{lll}
\hline Factor & Eigenvalue & \% of variance \\
\hline I & 10.35 & 79.6 \\
& Factor I
\end{tabular}

Self care:

0.81

A. Eating

B. Grooming

0.89

C. Bathing

0.87

D. Upper body dressing

0.88

E. Lower body dressing

F.Toiletting

0.93

Sphincter control:

0.89

G. Bladder management

H. Bowel management

Transfer:

0.94

I. Bed/chair/WC

J.Toilet

K.Tub/shower

Locomotion:

0.88

L.Walk/WC

M. Stairs

0.86

This picture differs slightly with the post surgery patients $(\mathrm{N}=56)$. There was a three factorial solution necessary for explaining $76 \%$ of the variance. The first factor contained FIM motor item, the second the cognitive items and the third one items referring to sphincter control and to eating. However, this was only the case in the FIM items on admission (Table 9) (Table 10). The items on discharge were again organized in two factorial solutions (75\% of variance explained). The most relevant factor contained cognitive items and items on sphincter control as well as on eating (Table 11). A factor analysis of the 13 motor items distinguished between motor items in a narrow sense (factor 1) and sphincter control, eating and grooming (factor 2). The solutions for the 13 FIM motor items on admission and on discharge are comparable (Table 12) (Table 13). 
Table 9 Factor analysis of 13 motor FIM items on discharge, vascular brain damage patients $(\mathrm{N}=75)$

\begin{tabular}{|c|c|c|c|}
\hline Factor & Eigenvalue & $\%$ of variance & cumulative \% \\
\hline \multirow[t]{2}{*}{ I } & 10.53 & 81 & \\
\hline & Factor I & & \\
\hline
\end{tabular}

Self care: $\quad 0.82$

\begin{tabular}{|c|c|}
\hline \multicolumn{2}{|l|}{ A. Eating } \\
\hline B. Grooming & 0.89 \\
\hline C. Bathing & 0.92 \\
\hline D. Upper body dressing & 0.89 \\
\hline E. Lower body dressing & 0.96 \\
\hline F.Toiletting & 0.95 \\
\hline $\begin{array}{l}\text { Sphincter } \\
\text { control: }\end{array}$ & 0.84 \\
\hline \multicolumn{2}{|l|}{ G. Bladder management } \\
\hline H. Bowel management & 0.82 \\
\hline Transfer: & 0.94 \\
\hline \multicolumn{2}{|l|}{ I. Bed/chair/WC } \\
\hline J.Toilet & 0.94 \\
\hline K. Tub/shower & 0.94 \\
\hline Locomotion: & 0.89 \\
\hline \multicolumn{2}{|l|}{ L.Walk/WC } \\
\hline M. Stairs & 0.89 \\
\hline
\end{tabular}

Table 10 Factor analysis of FIM on admission, post surgery patients $(\mathrm{N}=56)$

\begin{tabular}{llll}
\hline Factor & Eigenvalue & $\begin{array}{l}\text { \% of } \\
\text { variance }\end{array}$ & cumulative \% \\
\hline 1 & 10.56 & 58.7 & 70.4 \\
2 & 2.11 & 11.7 & \\
3 & 1.04 & 5.8 & 76.2 \\
\hline $\begin{array}{l}\text { Rotated factor } \\
\text { matrix (Varimax) }\end{array}$ & Factor I & Factor 2 & Factor 3 \\
\hline Self care: & 0.33 & 0.15 & 0.69
\end{tabular}

\begin{tabular}{|c|c|c|c|}
\hline A. Eating & & & \\
\hline B. Grooming & 0.51 & 0.2 & 0.67 \\
\hline C. Bathing & 0.7 & 0.12 & 0.5 \\
\hline $\begin{array}{l}\text { D. Upper body } \\
\text { dressing } .57\end{array}$ & & 0.21 & 0.61 \\
\hline $\begin{array}{l}\text { E. Lower body } \\
\text { dressing } .76\end{array}$ & & 0.26 & 0.32 \\
\hline F.Toiletting & 0.69 & 0.39 & 0.31 \\
\hline Sphincter control: & 0.25 & 0.36 & 0.69 \\
\hline $\begin{array}{l}\text { G. Bladder } \\
\text { management }\end{array}$ & & & \\
\hline $\begin{array}{l}\text { H. Bowel } \\
\text { management }\end{array}$ & 0.19 & 0.39 & 0.73 \\
\hline Transfer: & 0.87 & 0.26 & 0.22 \\
\hline I. Bed/chair/WC & & & \\
\hline J.Toilet & 0.86 & 0.31 & 0.21 \\
\hline K. Tub/shower & 0.85 & 0.24 & 0.3 \\
\hline Locomotion: & 0.63 & 0.28 & 0.44 \\
\hline L.Walk/WC & & & \\
\hline M. Stairs & 0.8 & 0.11 & 0.15 \\
\hline Communication: & 0.14 & 0.86 & 0.29 \\
\hline N. Comprehension & & & \\
\hline O. Expression & 0.21 & 0.82 & 0.27 \\
\hline Social cognition: & 0.22 & 0.86 & 0.04 \\
\hline P. Social interaction & & & \\
\hline Q. Problem solving & 0.36 & 0.74 & 0.3 \\
\hline R. Memory & 0.24 & 0.82 & 0.25 \\
\hline
\end{tabular}


Table II Factor analysis of FIM on discharge, post surgery patients $(\mathrm{N}=56)$

\begin{tabular}{llll}
\hline Factor & Eigenvalue & $\begin{array}{l}\% \text { of } \\
\text { variance }\end{array}$ & $\begin{array}{l}\text { cumulative } \\
\%\end{array}$ \\
\hline 1 & 11.63 & 64.6 & \\
2 & 1.94 & 10.8 & 75.4
\end{tabular}

\begin{tabular}{lll}
\hline $\begin{array}{l}\text { Rotated factor matrix } \\
\text { (Varimax) }\end{array}$ & Factor I & Factor $\mathbf{2}$ \\
\hline Self care: & 0.67 & 0.5
\end{tabular}

\section{A. Eating}

B. Grooming

0.59

0.55

C. Bathing

0.84

D. Upper body dressing .58

$$
0.58
$$

0.77

E. Lower body dressing .30

\section{F.Toiletting}

Sphincter control:

0.74

0.8

0.27

G. Bladder management

H. Bowel management

Transfer:

0.81

0.39

I. Bed/chair/WC

J.Toilet

K. Tub/shower

Locomotion:

L.Walk/WC

M. Stairs

Communication:

0.86

0.27

N. Comprehension

O. Expression

Social cognition:

0.92

0.2

P. Social interaction

Q. Problem solving

0.79

0.82
Table 12 Factor analysis of 13 motor FIM items on admission, post surgery patients $(\mathrm{N}=56)$

\begin{tabular}{llll}
\hline Factor & Eigenvalue & $\%$ of variance & $\begin{array}{l}\text { cumulative } \\
\%\end{array}$ \\
\hline I & 8.5 & 65.4 & 74 \\
2 & 1.12 & 8.6 & \\
\hline $\begin{array}{l}\text { Rotated factor } \\
\text { matrix (Varimax) }\end{array}$ & Factor I & Factor 2 \\
\hline $\begin{array}{l}\text { Self care: } \\
\text { n }\end{array}$ & 0.35 & 0.64
\end{tabular}

A. Eating

$\begin{array}{lll}\text { B. Grooming } & 0.53 \quad 0.66\end{array}$

$\begin{array}{lll}\text { C. Bathing } & 0.71 & 0.47\end{array}$

D. Upper body dressing $\quad 0.6 \quad 0.59$

E. Lower body dressing $\quad 0.78 \quad 0.39$

$\begin{array}{lll}\text { F.Toiletting } & 0.7 \mathrm{I} & 0.45\end{array}$

Sphincter control: $\quad 0.24 \quad 0.83$

G. Bladder

management

H. Bowel management $\quad 0.2 \quad 0.86$

$\begin{array}{lll}\text { Transfer: } & 0.88 & 0.29\end{array}$

I. Bed/chair/WC

J.Toilet

0.88

0.31

K.Tub/shower

0.87

0.35

Locomotion:

0.65

0.5

L.Walk/WC

M. Stairs

0.8

0.16 
Table 13 Factor analysis of 13 motor FIM items on discharge, post surgery patients $(\mathrm{N}=56)$

\begin{tabular}{llll}
\hline Factor & Eigenvalue & $\begin{array}{l}\% \text { of } \\
\text { variance }\end{array}$ & $\begin{array}{l}\text { cumulative } \\
\%\end{array}$ \\
\hline 1 & 8.67 & 66.7 & \\
2 & 1.22 & 9.4 & 76.1 \\
\hline
\end{tabular}

\begin{tabular}{lll}
\hline $\begin{array}{l}\text { Rotated factor } \\
\text { matrix (Varimax) }\end{array}$ & Factor I & Factor $\mathbf{2}$ \\
\hline Self care: & 0.37 & 0.82
\end{tabular}

A. Eating

\begin{tabular}{|c|c|c|}
\hline B. Grooming & 0.42 & 0.75 \\
\hline C. Bathing & 0.75 & 0.44 \\
\hline D. Upper body dressing & 0.45 & 0.74 \\
\hline E. Lower body dressing & 0.7 & 0.43 \\
\hline F.Toiletting & 0.75 & 0.48 \\
\hline Sphincter control: & 0.2 & 0.79 \\
\hline \multicolumn{3}{|l|}{$\begin{array}{l}\text { G. Bladder } \\
\text { management }\end{array}$} \\
\hline H. Bowel management & 0.27 & 0.84 \\
\hline Transfer: & 0.83 & 0.43 \\
\hline \multicolumn{3}{|l|}{ I. Bed/chair/WC } \\
\hline J.Toilet & 0.84 & 0.42 \\
\hline K.Tub/shower & 0.78 & 0.36 \\
\hline Locomotion: & 0.76 & 0.37 \\
\hline \multicolumn{3}{|l|}{ L.Walk/WC } \\
\hline M. Stairs & 0.82 & 0.07 \\
\hline
\end{tabular}

The FIM items of patients with rheumatologic illness $(\mathrm{N}=44)$ fit best in a two factor solution on admission and in a three factor solution on discharge (Table 14) (Table 15). Cognitive iItems and the item on bowel management build the first factor while the second factor consists of the motor items on admission. The FIM items on discharge show factor 1 with motor items, factor 2 with social cognition, bowel management and eating and factor 3 contains items on communication, explaining an additional $6 \%$ of variance. The factor analysis of the 13 motor items in patients with rheumatologic illness provides one factor solution on admission and 2 factor solutions on discharge where eating and bowel management required a second factor (Table 16) (Table 17).
Table I4 Factor analysis of FIM on admission, rheumatologic illness (N=44)

\begin{tabular}{|c|c|c|c|}
\hline Factor & Eigenvalue & $\begin{array}{l}\% \text { of } \\
\text { variance }\end{array}$ & $\begin{array}{l}\text { cumulative } \\
\%\end{array}$ \\
\hline I & 10.87 & 60.4 & \\
\hline 2 & 2.61 & 14.5 & 74.9 \\
\hline $\begin{array}{l}\text { Rotated factor matrix } \\
\text { (Varimax) }\end{array}$ & Factor I & Factor 2 & \\
\hline Self care: & 0.63 & 0.53 & \\
\hline \multicolumn{4}{|l|}{ A. Eating } \\
\hline B. Grooming & 0.64 & 0.57 & \\
\hline C. Bathing & 0.84 & 0.27 & \\
\hline $\begin{array}{l}\text { D. Upper body dressing } \\
.73\end{array}$ & & 0.44 & \\
\hline $\begin{array}{l}\text { E. Lower body dressing } \\
.79\end{array}$ & & 0.2 & \\
\hline F.Toiletting & 0.91 & 0.26 & \\
\hline Sphincter control: & 0.82 & 0.12 & \\
\hline \multicolumn{4}{|l|}{ G. Bladder management } \\
\hline H. Bowel management & 0.36 & 0.69 & \\
\hline Transfer: & 0.91 & 0.25 & \\
\hline \multicolumn{4}{|l|}{ I. Bed/chair/WC } \\
\hline J. Toilet & 0.89 & 0.23 & \\
\hline K.Tub/shower & 0.9 & 0.21 & \\
\hline Locomotion: & 0.92 & 0.11 & \\
\hline \multicolumn{4}{|l|}{ L.Walk/WC } \\
\hline M. Stairs & 0.79 & 0.33 & \\
\hline Communication: & 0.12 & 0.9 & \\
\hline
\end{tabular}

N. Comprehension

O. Expression

0.02

0.78

Social cognition:

0.19

0.72

P. Social interaction

Q. Problem solving

0.36

0.78

R. Memory

0.82 
Table I 5 Factor analysis of FIM on discharge, rheumatologic illness (N=44)

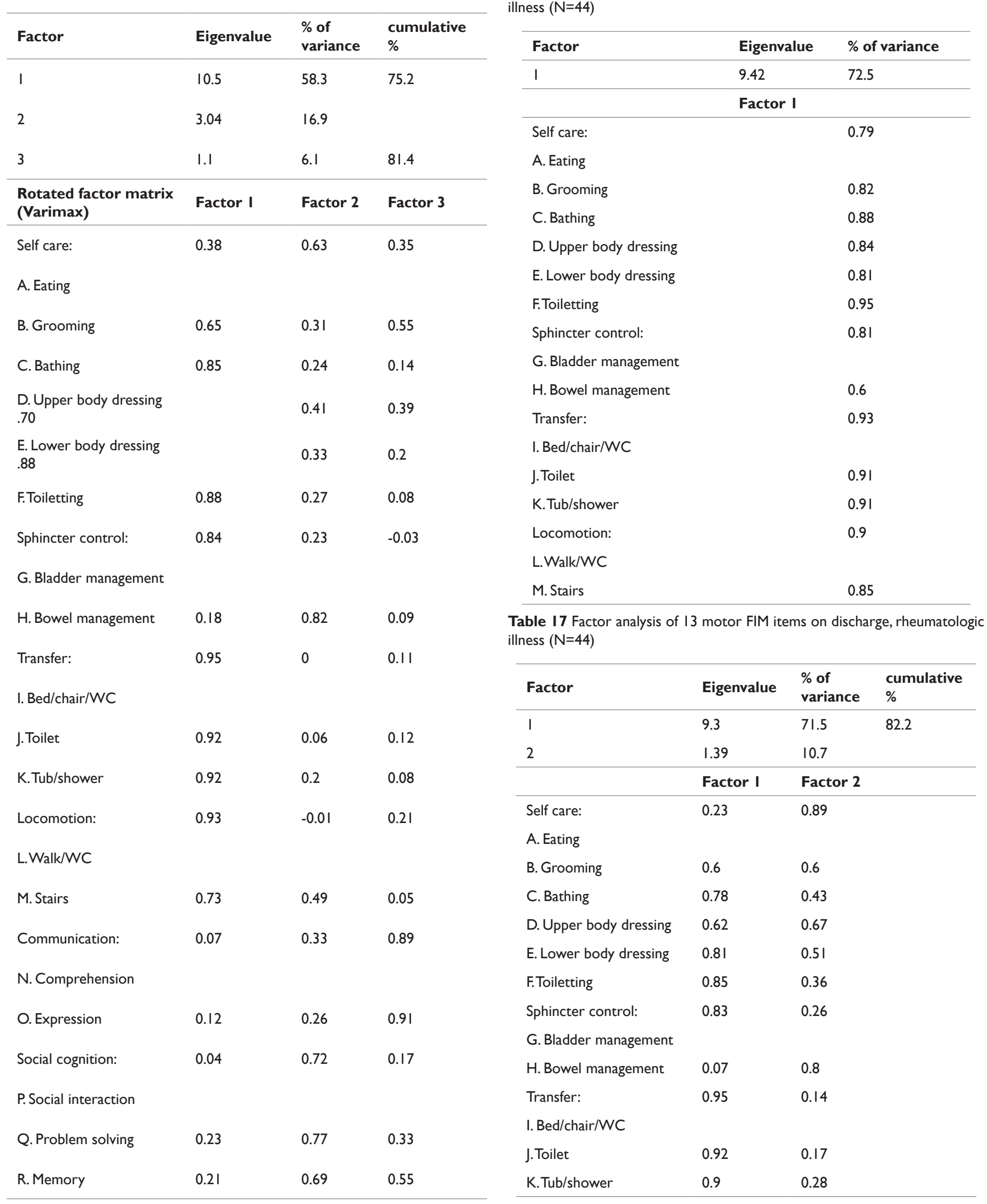

Table 16 Factor analysis of 13 motor FIM items on admission, rheumatologic iness $(\mathrm{N}=44)$

Table I 7 Factor analysis of 13 motor FIM items on discharge, rheumatologic 
Table continued...

\begin{tabular}{llll}
\hline Factor & Eigenvalue & $\begin{array}{l}\% \text { of } \\
\text { variance }\end{array}$ & $\begin{array}{l}\text { cumulative } \\
\%\end{array}$ \\
\hline $\begin{array}{l}\text { Locomotion: } \\
\text { L.Walk/WC }\end{array}$ & 0.91 & 0.21 \\
M. Stairs & 0.66 & 0.54 \\
\hline
\end{tabular}

The factor analysis of the residual group of patients with other neurological illnesses $(\mathrm{N}=46)$ provided 2 factor solution for FIM items on admission and on discharge, distinguishing between motor and cognitive items (Table 18) (Table 19). However, the 13 motor FIM items on admission required 2 factor solution (Factor 1: transfer items, sphincter control items as well as toliletting, walking on stairs and lower body dressing; Factor 2: grooming, eating, upper body dressing) (Tables 20-22).

Table 18 Factor analysis of FIM on admission, other neurological illnesses $(\mathrm{N}=46)$

\begin{tabular}{|c|c|c|c|}
\hline Factor & Eigenvalue & $\begin{array}{l}\% \text { of } \\
\text { variance }\end{array}$ & $\begin{array}{l}\text { cumulative } \\
\%\end{array}$ \\
\hline I & 10.47 & 58.2 & \\
\hline 2 & 3.31 & 18.4 & 76.5 \\
\hline $\begin{array}{l}\text { Rotated factor matrix } \\
\text { (Varimax) }\end{array}$ & Factor I & Factor 2 & \\
\hline Self care: & 0.63 & 0.43 & \\
\hline \multicolumn{4}{|l|}{ A. Eating } \\
\hline B. Grooming & 0.69 & 0.42 & \\
\hline C. Bathing & 0.84 & 0.24 & \\
\hline D. Upper body dressing .75 & & 0.45 & \\
\hline E. Lower body dressing .85 & & 0.23 & \\
\hline F. Toiletting & 0.83 & 0.21 & \\
\hline Sphincter control: & 0.8 & 0.34 & \\
\hline \multicolumn{4}{|l|}{ G. Bladder management } \\
\hline H. Bowel management & 0.76 & 0.24 & \\
\hline Transfer: & 0.89 & 0.14 & \\
\hline \multicolumn{4}{|l|}{ I. Bed/chair/WC } \\
\hline J.Toilet & 0.91 & 0.03 & \\
\hline K.Tub/shower & 0.92 & 0.06 & \\
\hline Locomotion: & 0.8 & 0.2 & \\
\hline \multicolumn{4}{|l|}{ L.Walk/WC } \\
\hline M. Stairs & 0.78 & -0.02 & \\
\hline Communication: & 0.15 & 0.92 & \\
\hline \multicolumn{4}{|l|}{ N. Comprehension } \\
\hline O. Expression & 0.17 & 0.91 & \\
\hline Social cognition: & 0.14 & 0.91 & \\
\hline
\end{tabular}

Table continued...

\begin{tabular}{lll}
\hline $\begin{array}{l}\text { Rotated factor matrix } \\
\text { (Varimax) }\end{array}$ & Factor I & Factor 2 \\
\hline P. Social interaction & & \\
Q. Problem solving & 0.24 & 0.87 \\
R. Memory & 0.19 & 0.93 \\
\hline
\end{tabular}

Table 19 Factor analysis of FIM on discharge, other neurological illnesses $(\mathrm{N}=46)$

\begin{tabular}{|c|c|c|c|}
\hline Factor & Eigenvalue & $\begin{array}{l}\% \text { of } \\
\text { variance }\end{array}$ & $\begin{array}{l}\text { cumulative } \\
\%\end{array}$ \\
\hline I & $10.7 \mid$ & 59.5 & 76.3 \\
\hline 2 & 3.02 & 16.8 & \\
\hline $\begin{array}{l}\text { Rotated factor } \\
\text { matrix (Varimax) }\end{array}$ & Factor I & Factor 2 & \\
\hline Self care: & 0.66 & 0.3 & \\
\hline \multicolumn{4}{|l|}{ A. Eating } \\
\hline B. Grooming & 0.77 & 0.33 & \\
\hline C. Bathing & 0.85 & 0.21 & \\
\hline $\begin{array}{l}\text { D. Upper body dressing } \\
.86\end{array}$ & & 0.33 & \\
\hline $\begin{array}{l}\text { E. Lower body dressing } \\
.85\end{array}$ & & 0.19 & \\
\hline F.Toiletting & 0.81 & 0.3 & \\
\hline Sphincter control: & 0.84 & 0.21 & \\
\hline \multicolumn{4}{|l|}{ G. Bladder management } \\
\hline H. Bowel management & 0.81 & 0.17 & \\
\hline Transfer: & 0.9 & 0.15 & \\
\hline \multicolumn{4}{|l|}{ I. Bed/chair/WC } \\
\hline J.Toilet & 0.92 & 0.13 & \\
\hline K.Tub/shower & 0.92 & 0.09 & \\
\hline Locomotion: & 0.77 & 0.23 & \\
\hline \multicolumn{4}{|l|}{ L.Walk/WC } \\
\hline M. Stairs & 0.79 & 0.06 & \\
\hline Communication: & 0.18 & 0.87 & \\
\hline \multicolumn{4}{|l|}{ N. Comprehension } \\
\hline O. Expression & 0.27 & 0.89 & \\
\hline Social cognition: & 0.14 & 0.88 & \\
\hline \multicolumn{4}{|l|}{ P. Social interaction } \\
\hline Q. Problem solving & 0.2 & 0.88 & \\
\hline R. Memory & 0.21 & 0.91 & \\
\hline
\end{tabular}


Table 20 Factor analysis of 13 motor FIM items on admission, other neurological illnesses $(\mathrm{N}=46)$

\begin{tabular}{llll}
\hline Factor & Eigenvalue & $\begin{array}{l}\% \text { of } \\
\text { variance }\end{array}$ & $\begin{array}{l}\text { cumulative } \\
\%\end{array}$ \\
\hline 1 & 9.17 & 70.5 & \\
2 & 1.14 & 8.7 & 79.3
\end{tabular}

\begin{tabular}{lll}
\hline $\begin{array}{l}\text { Rotated factor } \\
\text { matrix (Varimax) }\end{array}$ & Factor I & Factor $\mathbf{2}$ \\
\hline Self care: & 0.24 & 0.85
\end{tabular}

\begin{tabular}{|c|c|c|}
\hline \multicolumn{3}{|l|}{ A. Eating } \\
\hline B. Grooming & 0.28 & 0.9 \\
\hline C. Bathing & 0.57 & 0.7 \\
\hline $\begin{array}{l}\text { D. Upper body } \\
\text { dressing }\end{array}$ & 0.41 & 0.84 \\
\hline $\begin{array}{l}\text { E. Lower body } \\
\text { dressing }\end{array}$ & 0.68 & 0.56 \\
\hline F.Toiletting & 0.75 & 0.43 \\
\hline Sphincter control: & 0.77 & 0.43 \\
\hline \multicolumn{3}{|l|}{$\begin{array}{l}\text { G. Bladder } \\
\text { management }\end{array}$} \\
\hline $\begin{array}{l}\text { H. Bowel } \\
\text { management }\end{array}$ & 0.7 & 0.41 \\
\hline Transfer: & 0.81 & 0.42 \\
\hline \multicolumn{3}{|l|}{ I. Bed/chair/WC } \\
\hline J.Toilet & 0.85 & 0.36 \\
\hline K.Tub/shower & 0.85 & 0.38 \\
\hline Locomotion: & 0.56 & 0.62 \\
\hline \multicolumn{3}{|l|}{ L.Walk/WC } \\
\hline M. Stairs & 0.84 & 0.13 \\
\hline
\end{tabular}

Table 2 I Factor analysis of 13 motor FIM items on discharge, other neurological illnesses $(\mathrm{N}=46)$

\begin{tabular}{|c|c|c|}
\hline Factor & Eigenvalue & $\%$ of variance \\
\hline \multirow[t]{2}{*}{ I } & 9.5 & 73.1 \\
\hline & Factor I & \\
\hline Self care: & & 0.72 \\
\hline \multicolumn{3}{|l|}{ A. Eating } \\
\hline B. Grooming & & 0.83 \\
\hline C. Bathing & & 0.87 \\
\hline D. Upper body dressing & & 0.92 \\
\hline E. Lower body dressing & & 0.87 \\
\hline F.Toiletting & & 0.86 \\
\hline Sphincter control: & & 0.86 \\
\hline \multicolumn{3}{|l|}{ G. Bladder management } \\
\hline H. Bowel management & & 0.83 \\
\hline Transfer: & & 0.9 \\
\hline \multicolumn{3}{|l|}{ I. Bed/chair/WC } \\
\hline J.Toilet & & 0.92 \\
\hline K.Tub/shower & & 0.91 \\
\hline Locomotion: & & 0.8 \\
\hline \multicolumn{3}{|l|}{ L.Walk/WC } \\
\hline M. Stairs & & 0.78 \\
\hline
\end{tabular}

Table 22 The percentage of variance explained by the first factor

\begin{tabular}{|c|c|c|c|c|}
\hline Sample & admission & $\begin{array}{l}\text { all items } \\
\text { discharge }\end{array}$ & $\begin{array}{l}\text { motor } \\
\text { items } \\
\text { admission }\end{array}$ & discharge \\
\hline All patients & 65 & 65 & 75 & 76 \\
\hline $\begin{array}{l}\text { Vascular brain } \\
\text { damage }\end{array}$ & 70 & 69 & 80 & 81 \\
\hline Post surgery & 59 & $65^{*}$ & 65 & 67 \\
\hline $\begin{array}{l}\text { Rheumatologic } \\
\text { illness }\end{array}$ & 60 & 58 & 72 & 72 \\
\hline $\begin{array}{l}\text { Other } \\
\text { neurological } \\
\text { illness }\end{array}$ & 58 & 60 & 71 & 73 \\
\hline
\end{tabular}




\section{Discussion}

The issue of unidimensionality of FIM as of any other tests is a serious one. The experienced methodologist will see a set of items presented as a test leading to a single score, as a system with items, subgroups and a total if every assumption is fulfilled. This is seldom the case but mostly adopted for pragmatic purposes. However, when these shortcuts become a research entity referring not to other research data but for classifying individuals, groups and their changes problems arise. Obviously, functional independence is not a construct that could be built up through addition of requirements only. It is a phenomenological and experiential unit equally contributed to by the social environmental features of the target individual embedding. But this is not the issue here. Performing a series of factor analyses we can also confirm the dimensionality of FIM. Primarily, it is the distinction of the motor and cognitive items, but also other items, if specific groups of patients are considered. As indicated by Ravaud et al. ${ }^{1}$ sphincter control is often separated as another dimension and the items in self care seem to be less than monolithic as often seen in different factors such as eating, grooming on one hand, dressing lower body and toiletting on the other. However, it should also be recognized that factor 1 explains a higher proportion of the variance, in our case varying between 58 and $81 \%$. If FIM is used for assessment, we would recommend that dimensional analysis is used before any summative scores are used. Further, the FIM results should be used in a multiple way thus addressing the item level, groups of items that should be empirically supported by data in evaluation as well as by other normative data.

Consequently, as the results do not only refer to the elements of a data set but also to the relationship between the data, any changes in dimensionality are also results worth reporting in which the appropriate multidimensional scaling methods should be used in analysis. Finally, as indicated in our data, the dimensionality is not given for all patients and it is possible to propose that some of the additional dimensionality found in the study by Ravaud et al. ${ }^{1}$ may be due to a different cultural outfit of the patients and the assessors even if the translation of usage is as close as possible to the original intentions. We should not forget that FIM is dealing with social or socially relevant and meaningful behaviour that often is culturally specific. Summarizing the argument here it could be said that care in dealing with the FIM data can help us in providing meaningful results. However, a conceptual creativity in designing new instruments assessing concepts such as independence should be encouraged. ${ }^{2}$

\section{Acknowledgments}

None.

\section{Conflict of interest}

The authors declare no conflict of interest.

\section{References}

1. Ravaud JF, Delcey M, Yelnik A. Construct validity of the functional independence measure (fim): questioning the unidimensionality of the scale and the 'value of fim scores. Scand J Rehabil Med. 1999;31(1):3141 .

2. Scheuringer M, Grill E, Boldt C, et al. Latent class factor analysis of the functional independence measure confirmed four distinct dimensions in patients undergoing neurological rehabilitation. Eur J Phys Rehabil Med. 2011;47(1):25-34. 